\title{
Redescripción de Parodon caliensis y Saccodon dariensis (Characiformes: Parodontidae)
}

\author{
Alejandro Londoño-Burbano \& César Román-Valencia \\ Universidad del Quindío, Laboratorio de Ictiología, A. A. 2639, Armenia, Colombia; ceroman@uniquindio.edu.co \\ Recibido 09-VIII-2009. Corregido 12-I-2010. Aceptado 15-II-2010.

\begin{abstract}
Redescription of Parodon caliensis and Saccodon dariensis (Characiformes: Parodontidae). Parodontidae family is a group of Characiformes fishes distributed throughout South America and parts of Panama, except in the basins of the southern Bahia state in Brasil on the Atlantic coast, Patagonia and the Amazon river channel. The family includes three genera: Apareiodon Eigenmann 1916, Parodon Valenciennes 1849 y Saccodon Kner 1863, 28 recognized species and two valid genera: Parodon and Saccodon. Redescription of Parodon caliensis and Saccodon dariensis is carried out based on type, and topotypic material from Colombia. Significant differences were found in morphometric, meristic, osteologic and color characters. S. dariensis is widely distributed but $P$. caliensis is restricted to the upper Cauca River drainage. Three species are considered herein as synonyms of $S$. dariensis: Apareiodon dariensis, A. compressus and $S$. caucae. Sexual dimorphism is described for both species. Rev. Biol. Trop. 58 (3): 813-826. Epub 2010 September 01.
\end{abstract}

Key words: Parodon, Saccodon, taxonomy, Colombia, tropical fish.

La familia Parodontidae es un grupo de peces Characiformes distribuidos a través de Sur América y parte de Panamá, excepto en las cuencas del sur del Estado de Bahía en Brasil en la costa Atlántica, la Patagonia, y el canal del Río Amazonas (Pavanelli 2003). Es interesante resaltar su ausencia en el canal amazónico, lo que evidencia un carácter de ramoneadores (Pavanelli 1999). De acuerdo a Pavanelli (1999, 2003) contiene tres géneros: Apareiodon Eigenmann 1916, Parodon Valenciennes 1849 y Saccodon Kner 1863, que incluyen 28 especies reconocidas y dos géneros válidos: Parodon y Saccodon. Apareiodon se considera sinónimo junior de Parodon (Ingenito 2008).

Los géneros Parodon y Saccodon se reconocen por la presencia de un radio no ramificado en la aleta pectoral (contra dos radios no ramificados) y la presencia de dientes laterales en la mandíbula inferior (contra ausencia), los cuales son informativos en taxonomía (Pavanelli 1999) y en filogenia (Ingenito 2008). Ambos táxones son monofiléticos (Ingenito 2008).

Eigenmann (1922), Miles (1943, 1947), Dahl (1971), Mojica et al. (2002), Maldonado et al. (2005) reportaron a Parodon caliensis presente en el sistema del Magdalena, mientras que Londoño-Burbano (2009) lo encuentra sólo en la porción del alto Cauca. Eigenmann (1922), Miles (1943, 1947), Dahl (1971), Mojica et al. (2002), Maldonado et al. (2005) al mencionar $P$. caliensis, ilustraron ejemplares con el modelo de coloración propio de Saccodon dariensis, lo cual ha llevado a un sinnúmero de confusiones en la taxonomía de ambas especies. Mojica et al. (2002) reportan ambas especies como endémicas y casi amenazadas. Por lo tanto, el objetivo de este trabajo es efectuar la redescripción de Parodon caliensis y Saccodon dariensis. 


\section{MATERIAL Y MÉTODOS}

Las mediciones y conteos se realizaron según Pavanelli \& Britski (2003). Los datos se tomaron con ayuda de un estereoscopio, aguja y calibrador digital de precisión $0.01 \mathrm{~mm}$, del lado izquierdo de los ejemplares a excepción de cuando estaban deteriorados. Se utilizó el programa Scion Image bajo Windows para la toma de datos morfométricos y merísticos a partir de fotografías de material tipo y no tipo cuando no fue posible examinar material directamente. Se realizó un análisis exploratorio de componentes principales (ACP) por medio del programa Past versión 1.52 bajo Windows para los datos morfométricos entre las poblaciones y entre las especies, de la siguiente manera: 1) un análisis comparado entre las poblaciones de los afluentes con mayor proximidad geográfica y 2) entre las especies de los principales sistemas hidrográficos analizados. Se aplicó la técnica de corrección alométrica (Burnaby 1966) para corregir el efecto de la talla en dicho análisis. Las medidas se consignaron (Cuadro 1) como porcentajes de longitud estándar (LE) y cabeza (LC); las proporciones, conteos, rangos y desviación estándar de la media, se calcularon con ayuda del programa Microsoft Excel (2007). El registro de radios en las aletas es: números romanos en minúscula para radios no ramificados y arábigos para ramificados (Cuadro 2). Para el análisis de los conteos se realizaron cajas de Tukey o box plots, se tomó la media aritmética como medida de tendencia central. En merística, para los valores máximos y mínimos se asumió la normalidad en los datos, usando el programa Past versión 1.52 bajo Windows. Se realizaron observaciones osteológicas a ejemplares clareados y teñidos, según Taylor \& Van Dyke (1985) modificado por Song \& Parenti (1995). La nomenclatura osteológica sigue a Weitzman (1962), Roberts (1974a, b) e Ingenito (2008); para la traducción de nombres al castellano se siguió a Ruiz-Calderón \& Román-Valencia (2006). Los conteos de vértebras en $P$. caliensis se realizaron a partir de radiografías de la serie tipo de la especie (lectotipo y cuatro paralectotipos). La evaluación del polimorfismo dentario en $S$. dariensis relacionada con los morfos I-V se basó en Roberts (1974a).

El material examinado para las especies se citó así: acronimia de la institución de la cual forma parte el lote con su respectivo número de colección, número de ejemplares examinados y consignados en los cuadros escritos en números arábigos, al incluir examen de material diafanizado (C\&T), se consignó la cantidad de ejemplares en número arábigos, con el rango de mayor y menor longitud estándar (LE) en mm de cada lote, país y sistema al que pertenece por ejemplo: BMNH 1895.11.16.83 (57mm LE) Colombia, cuenca del Río Cauca. La información que se encuentra en el lote se citó de la siguiente manera: nombre del país, localidad, departamento (o en su defecto estado o provincia) y fecha de colecta.

El material examinado en este estudio pertenece a instituciones de diversos países. En los Estados Unidos: American Museum of Natural History, Nueva York (AMNH), California Academy of Sciences, San Francisco (CAS), Field Museum of Natural History, Chicago (FMNH), Museum of Comparative Zoology, Cambridge (MCZ). En el Reino Unido: British Museum of Natural History, Londres $(\mathrm{BMNH})$. En Colombia: Colección de peces de la Universidad Católica de Oriente, Río Negro (CP-UCO), Instituto para la investigación y preservación del patrimonio cultural y natural del Valle del Cauca, INCIVA, Cali (IMCN), y Laboratorio de Ictiología, Universidad del Quindío, Armenia (IUQ). Material de comparación examinado en Londoño-Burbano (2009).

\section{RESULTADOS}

Parodon caliensis Boulenger, 1895 (Cuadros 1, 2 y Fig. 1)

Parodon caliensis Boulenger 1895: 480 (Localidad tipo: Rio Cali, cuenca del Río Cauca, Colombia). Eigenmann 1922: 108109 (clave, datos merísticos y morfométricos, distribución, ilustración en vista lateral que 
CUADRO 1

Datos morfométricos de $\mathrm{P}$. caliensis $y \mathrm{~S}$. dariensis

TABLE 1

Morphometric data of $\mathrm{P}$. caliensis and $\mathrm{S}$. dariensis

\begin{tabular}{|c|c|c|c|c|c|c|c|c|}
\hline & \multicolumn{4}{|c|}{ P. caliensis } & \multicolumn{4}{|c|}{ S. dariensis } \\
\hline & Promedio & Menor & Mayor & $\mathrm{DE}$ & Promedio & Menor & Mayor & $\mathrm{DE}$ \\
\hline Longitud estándar & 66.41 & & & & 64.52 & & & \\
\hline Longitud total & 80.58 & & & & 80.48 & & & \\
\hline \multicolumn{9}{|l|}{ Porcentajes en longitud estándar } \\
\hline Altura del cuerpo & 27.32 & 23.69 & 32.34 & 2.43 & 15.03 & 6.65 & 37.07 & 2.23 \\
\hline Altura del pedúnculo caudal & 12.61 & 11.2 & 14.57 & 1.25 & 7.3 & 3.25 & 17.92 & 0.9 \\
\hline Longitud aleta anal & 15.26 & 14.19 & 16.31 & 0.66 & 10.71 & 5.61 & 25.67 & 1.29 \\
\hline Longitud de la base de la aleta dorsal & 11.82 & 10.95 & 12.54 & 0.45 & 7.88 & 4.11 & 17.54 & 0.77 \\
\hline Longitud de la cabeza & 23.91 & 21.21 & 25.73 & 1.51 & 13.73 & 7.97 & 27.39 & 2.18 \\
\hline Longitud aleta dorsal & 21.55 & 20.84 & 22.29 & 0.44 & 14.44 & 8.23 & 33.26 & 1.59 \\
\hline Longitud aleta pectoral & 21.14 & 19.64 & 23.1 & 1.01 & 14.81 & 8.38 & 32.73 & 1.75 \\
\hline Longitud aleta pélvica & 17.67 & 15.44 & 19.46 & 1.05 & 11.87 & 6.7 & 25.83 & 1.56 \\
\hline Longitud del pedúnculo caudal & 14.23 & 12.56 & 17.23 & 1.45 & 8.05 & 3.58 & 18.36 & 0.99 \\
\hline Longitud predorsal & 49.23 & 46.86 & 51.02 & 1.29 & 30.66 & 16.12 & 67.51 & 1.23 \\
\hline Distancia entre la adiposa y la anal & 17.19 & 15.74 & 18.48 & 0.71 & 10.35 & 4.47 & 25.23 & 1.19 \\
\hline Distancia entre la anal y el final de la dorsal & 29.03 & 26.88 & 32.52 & 1.49 & 19.93 & 9.33 & 50.84 & 2.63 \\
\hline Distancia entre la dorsal y la pélvica & 27.05 & 23.56 & 31.87 & 2.64 & 14.93 & 6.89 & 37.31 & 2.54 \\
\hline Distancia entre la dorsal y el supraoccipital & 30.78 & 28.67 & 33.13 & 1.16 & 18.27 & 8.89 & 43.1 & 1.61 \\
\hline Distancia entre la pectoral y la dorsal & 33.51 & 31.16 & 36.48 & 1.35 & 20.64 & 9.12 & 50.06 & 2.33 \\
\hline Distancia entre la pectoral y la pélvica & 32.8 & 29.63 & 37.8 & 2.57 & 21.64 & 9.64 & 52,00 & 2.75 \\
\hline Distancia entre la pectoral y el final de la dorsal & 41.31 & 38.28 & 45.3 & 1.84 & 26.03 & 11.92 & 61.72 & 2.65 \\
\hline Distancia entre la pélvica y la adiposa & 36.28 & 34.2 & 43.35 & 2.41 & 22.94 & 10.35 & 53.32 & 1.94 \\
\hline Distancia entre la pélvica y la anal & 25.95 & 23.33 & 29.56 & 1.87 & 17.73 & 8.34 & 42.64 & 2.32 \\
\hline Distancia entre la pélvica y el final de la dorsal & 23.68 & 21,00 & 28.81 & 2.54 & 12.84 & 5.65 & 31.59 & 1.93 \\
\hline Distancia entre el final de la dorsal y la adiposa & 26.38 & 24.1 & 32.56 & 1.84 & 18.39 & 8.76 & 44.84 & 2.26 \\
\hline Distancia entre el istmo y la dorsal & 37.83 & 35.01 & 47.15 & 3.08 & 23.24 & 10.15 & 53.88 & 1.97 \\
\hline \multicolumn{9}{|l|}{ Porcentajes en longitud de la cabeza } \\
\hline Longitud del hocico & 33.75 & 25.08 & 38.04 & 4.02 & 4.41 & 2.4 & 9.08 & 2.72 \\
\hline Diámetro del ojo & 24.25 & 21.09 & 26.71 & 1.92 & 3.3 & 2.52 & 5.23 & 4.32 \\
\hline Distancia entre la unión maxilar y el istmo & 44.4 & 38.33 & 48.54 & 2.58 & 6.11 & 4.24 & 10.81 & 9.1 \\
\hline Distancia entre el hocico y la unión maxilar & 26.57 & 20.56 & 29.78 & 2.41 & 4.2 & 1.92 & 9.29 & 6.24 \\
\hline Distancia entre el hocico y el istmo & 68.22 & 53.31 & 73.98 & 4.97 & 9.97 & 6.12 & 19.36 & 8.98 \\
\hline Distancia entre el hocico y el supraoccipital & 75.52 & 54.52 & 88.12 & 10.95 & 12.66 & 7.69 & 25.3 & 2.89 \\
\hline Distancia entre el istmo y la pectoral & 33.53 & 30.55 & 36.92 & 2.35 & 5.26 & 1.93 & 12.95 & 7.17 \\
\hline Distancia entre el supraoccipital y la unión maxilar & 73.86 & 68.42 & 86.16 & 5.07 & 10.62 & 5.64 & 21.01 & 5.44 \\
\hline Distancia entre el supraoccipital y la pectoral & 74.33 & 68.69 & 82.96 & 4.31 & 9.78 & 5.04 & 21.37 & 6.34 \\
\hline Distancia entre el supraoccipital y el istmo & 69.72 & 61.36 & 82.65 & 5.98 & 9.31 & 5.15 & 19.09 & 3.05 \\
\hline Distancia interorbital & 40.12 & 37.96 & 42.91 & 1.47 & 5.87 & 2.71 & 13.97 & 5.69 \\
\hline Longitud de la mandíbula & 20.67 & 18.49 & 22.35 & 1.09 & 2.43 & 1.49 & 4.36 & 1.67 \\
\hline
\end{tabular}

$\mathrm{DE}=$ desviación estándar.

$\mathrm{DE}=(\mathrm{SD})$ standard deviation. 
CUADRO 2

Conteos merísticos de $\mathrm{P}$. caliensis y $\mathrm{S}$. dariensis

TABLE 2

Meristic counts of $\mathrm{P}$. caliensis and $\mathrm{S}$. dariensis

\begin{tabular}{lcccccc} 
& \multicolumn{3}{c}{ S. dariensis } \\
& Menor & Mayor & DE & Menor & Mayor & DE \\
Cúspides de los dientes del premaxilar & 13 & 15 & 1.41 & 0 & 20 & 7.7 \\
Dientes del maxilar & 1 & 2 & 0.7 & 0 & 2 & 0.82 \\
Dientes del premaxilar & 4 & 5 & 0.7 & 3 & 4 & 0.53 \\
Dientes del dentario & 1 & 2 & 0.7 & 0 & 0 & 0,00 \\
Escamas circumpedunculares & 15 & 16 & 0.7 & 12 & 12 & 0,00 \\
Escamas de la línea lateral & 39 & 40 & 0.7 & 38 & 41 & 1.51 \\
Escamas entre la dorsal y la adiposa & 11 & 14 & 2.12 & 14 & 15 & 0.48 \\
Escamas posadiposa & 6 & 8 & 1.41 & 6 & 8 & 0.67 \\
Escamas posanales & 1 & 2 & 0.7 & 6 & 10 & 1.34 \\
Escamas preanales & 26 & 29 & 2.12 & 22 & 29 & 2.3 \\
Escamas predorsales & 12 & 15 & 2.12 & 12 & 14 & 0.82 \\
Escamas de la línea transversal debajo de la línea lateral & 4 & 5 & 0.71 & 3 & 5 & 0.63 \\
Escamas de la línea transversal encima de la línea lateral & 4 & 5 & 0.71 & 4 & 5 & 0.42 \\
Radios de la aleta anal & $\mathrm{i}, 6$ & $\mathrm{i}, 7$ & 0.71 & $\mathrm{ii}, 6$ & $\mathrm{i} .9$ & 1.41 \\
Radios de la aleta caudal & $\mathrm{i}, 17, \mathrm{i}$ & $\mathrm{i}, 17, \mathrm{i}$ & 0,00 & $\mathrm{i}, 17, \mathrm{i}$ & $\mathrm{i} .19 . \mathrm{i}$ & 1.41 \\
Radios de la aleta dorsal & $\mathrm{ii}, 9$ & $\mathrm{ii}, 10$ & 0.71 & $\mathrm{ii}, 9$ & $\mathrm{ii}, 10$ & 0.71 \\
Radios de la aleta pectoral & $\mathrm{i}, 12$ & $\mathrm{i}, 13$ & 0.71 & $\mathrm{ii}, 15$ & $\mathrm{i}, 16$ & 0.71 \\
Radios de la aleta pélvica & $\mathrm{i}, 7$ & $\mathrm{i}, 7$ & 0,00 & $\mathrm{i}, 7, \mathrm{i}$ & $\mathrm{i}, 8, \mathrm{i}$ & 0.71
\end{tabular}

$\mathrm{DE}=$ desviación estándar.

$\mathrm{DE}=(\mathrm{SD})$ standard deviation.

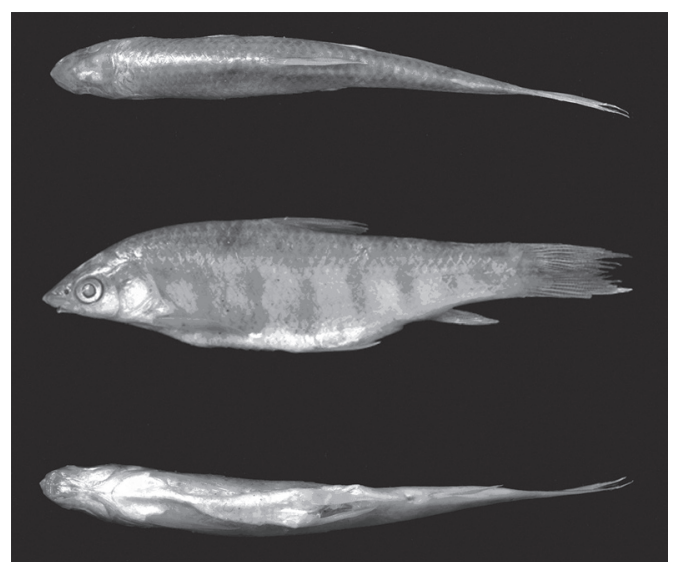

Fig. 1. Vistas dorsal, lateral y ventral de Parodon caliensis, BMNH 1895.11.16.83, lectotipo, 57mm LE. Fotos por J. Maclaine (BMNH).

Fig. 1. Dorsal, lateral and ventral views of Parodon caliensis, BMNH 1895.11.16.83, lectotype, 57mm SL. Photos by J. Maclaine (BMNH). corresponde a $S$. dariensis). Miles 1943: 42 (descripción, distribución, conteos de escamas). Miles 1947: 131-132 (clave, distribución, conteos de escamas sobre la línea lateral y talla máxima). Roberts 1974b: 424-434; 469 (osteología de la familia, relaciones con respecto a Hemiodontidae, ilustración de la mandíbula superior e inferior). Géry 1977: 203 (clave, conteos de escamas, localidad tipo). Mojica et al. 2002: 209 (datos taxonómicos, endémica del alto Cauca, casi amenazada). Pavanelli 1999: 183-189 (revisión de la familia, coloración, distribución, notas ecológicas, datos morfométricos y merísticos). Pavanelli 2003: 46-47 (sinonimias, distribución, localización de sintipos, talla máxima, nombres comunes). Maldonado et al. 2005: 38; 255; 301 (ilustración en vista lateral que corresponde a $S$. dariensis), sinonimias, categoría de conservación, 
datos sobre ecología, distribución y registros en colecciones. Ingenito 2008: 13 (análisis filogenético).

Parodon calienses Dahl 1971: 117 (clave, distribución, ilustración en vista lateral que corresponde a $S$. dariensis).

\section{Material Examinado}

Lectotipo. BMNH 1895.11.16.83 (57 mm LE) Colombia, cuenca del Río Cauca. Colombia; River: Cali. Cali River, Colombia, alt. 3 200ft. Departamento del Valle del Cauca.

Paralectotipos. BMNH 1895.11.16.84-87 (4: 48-59mm LE) colectados con el lectotipo.

No tipos. Todos de Colombia: IUQ 2626 (1 C\&T: 83.08mm LE), río Cauca en Decepaz, Cali, Departamento del Valle del Cauca, 4-II1999. CAS 6779 (5: 48.23-56.01mm de LE) Colombia, cuenca del Río Cauca. Sur América, Colombia, Valle del Cauca. R. Magdalena basin, R. Cauca, Cali, elev. 3 312ft. Departamento del Valle del Cauca, 1-III-1912. CAS 6780 (3: 82.18-89.53mm de LE), cuenca del Río Cauca. Sur América, Colombia, Cauca. Magdalena R. basin, Río Cauca basin. Cartago en Rio La Vieja, seis millas del rio Cauca; 3 012ft. elev. Departamento del Valle del Cauca, 22-II-1912 al 23-II-1912. AMNH 7110 (2: 60.53 y $64.98 \mathrm{~mm}$ LE), cuenca del Río Cauca. Sur América, Colombia, Valle, Rio Cauca, Cali Departamento del Valle del Cauca, 1-IV- 1912. FMNH 69790 (6: 53.23- 62.02mm LE), cuenca del Río Cauca. Sur América, Río Cauca en Cali, Departamento del Valle del Cauca, 1-III1912. FMNH 56305 (4: 83.10-103.19mm LE), cuenca del Río Cauca. Sur América, Colombia, municipio de Cartago Departamento del Valle del Cauca, 22-II-1912. MCZ 35893 (2: 50.40 y $58.09 \mathrm{~mm}$ de LE), cuenca del Río Cauca. Alto Rio Cauca and tributaros, Valle del Cauca, Colombia, Sur América Cali, X-1942.

English diagnosis. $P$. caliensis differs from $S$. dariensis by having one unbranched pectoral-fin ray (vs. two unbranched pectoral. fin rays), by the presence of lateral teeth in the lower jaw (vs. absence), by having vertical bands without horizontal stripe (vs. round spots, set apart, in three rows along the body) and by the circum-peduncle scales (16 vs. 12). $P$. caliensis is distinguished from its congeners from the presence of six to eight vertical bars, two to three scales wide, along the body flanks (vs. absence, except in P. apolinari, which has 14-17 vertical bars one to two scales wide), by the absence of a lateral stripe (vs. a lateral stripe with upwards and downwards projections, except in $P$. apolinari) and by all the fins being hyaline except the caudal fin (vs. pectoral and pelvic fins with cromatophores or dark spots).

Diagnosis. $P$. caliensis se diferencia de $S$. dariensis por presentar un radio no ramificado en la aleta pectoral (contra dos radios no ramificados); con dientes laterales en la mandíbula inferior (contra ausencia); bandas verticales sin franja horizontal (contra manchas redondas, separadas, en tres filas a lo largo del cuerpo); número de escamas circumpedunculares (16 contra 12). De sus congéneres por presentar de 6-8 bandas verticales de 2-3 escamas de ancho a lo largo de su porción lateral (contra ausencia, excepto P. apolinari que presenta de 14-17 bandas verticales más angostas, de 1-2 escamas de ancho); por la ausencia de franja lateral horizontal (contra presencia de franja lateral con proyecciones hacia arriba y hacia abajo, excepto en $P$. apolinari) y todas las aleta hialinas, excepto la caudal (contra aletas pareadas y axiales con cromatóforos o manchas de color negro, marrón o gris).

Descripción. Datos morfométricos y merísticos se consignaron en los cuadros 1 y 2 , respectivamente. Cuerpo rollizo. Perfil dorsal convexo desde el hocico hasta el inicio de la aleta dorsal, a partir de allí en línea recta hasta el inicio de la aleta adiposa y cóncavo hasta el inicio del lóbulo caudal superior. Silueta ventral de la cabeza recta desde el hocico hasta una vertical trazada con el inicio del tercer infraorbital, a partir de allí y hasta el final del 
istmo, redondeada. Istmo ubicado en la vertical trazada en la parte anterior del opérculo, membranas branquiales unidas entre si y libres del istmo. Ojos laterales; boca ventral, labio superior ausente. Perfil ventral del cuerpo redondeado desde el istmo hasta el inicio de la aleta anal y de allí recto hasta el inicio del lóbulo caudal inferior. Pedúnculo caudal comprimido. Hemiserie del premaxilar con cuatro dientes, dientes con el borde inferior recto, con 12-15 cúspides pequeñas, encajan con la mandíbula inferior. Esta con 1-3 dientes laterales, más grandes, sin cúspides, curvos hacia afuera, que no se observan cuando la boca está cerrada. Maxila con 1-2 dientes, más pequeños que los presentes en el premaxilar, multicuspides, su mitad insertada en el tejido que recubre esta porción. Aletas pectorales cortas, no alcanzan las aletas ventrales ni una vertical trazada desde su parte más distal hasta el inicio de la aleta dorsal, su inicio en una vertical trazada hasta una escama posterior al final del supraoccipital, truncadas, el cuarto y quinto radio ramificado mas alargados que el resto. Aletas ventrales truncadas, no alcanzan el poro genital por dos o tres escamas, su origen en una vertical al extremo posterior de la aleta dorsal o una escama anterior a este, primer y segundo radio ramificado más largos. Aleta anal truncada, primero y segundo radio ramificados más alargados. Aleta dorsal truncada, con el primer y segundo radio ramificado más alargado que el resto, su inicio en una vertical hasta la mitad de la distancia entre las cinturas pectoral y ventral, no alcanza la aleta adiposa por más de seis escamas. Origen de la aleta adiposa en una vertical desde el extremo posterior de la aleta anal. Aleta caudal bilobulada, ambos lóbulos de igual tamaño, radios medios de color marrón claro hasta el extremo distal, base escamada hasta su tercera parte. Línea lateral completa con 39-40 escamas con poros, el canal y puede extenderse hasta la tercera parte de esta.

Observaciones osteológicas. Antorbital triangular, parte ventral ancha, se adelgaza hacia el extremo dorsal, parte media en contacto con el infraorbital uno; parte dorsal no está en contacto con el supraorbital, el cual presenta un ancho continuo en toda su longitud, en contacto con el sexto infraorbital. Nasal convexo en la superficie ventral, en contacto posteriormente con la parte anterior del frontal. Premaxilar poco desarrollado en comparación con $S$. dariensis, dientes de reposición insertada en la porción dorsal. Maxilar muy grueso en su parte posterior, más delgada en el segmento medio, mientras que tanto su porción ventral como dorsal se observan rectas. Dientes del premaxilar y maxilar multicúspides. Postemporal igual de ancho en toda su longitud, parte anteroventral en contacto con el borde posterior del extraescapular. Pterótico de forma irregular, extremo anterior en contacto con el sexto infraorbital. Metapterigoides grande, unido por cartílago al cuadrado, la anterior en contacto con el ectopterigoides y el mesopterigoides, éste último de mayor tamaño que en $S$. dariensis donde es alargado, mientras en $P$. caliensis es dos veces más grueso, redondo en la parte dorsal y recto en la parte ventral. Ectopterigoides largo y delgado, de forma regular. Palatino de forma muy irregular: superficie posterodorsal con una porción cartilaginosa la cual lo une a la porción ventral del mesopterigoides, parte anterior en contacto con el vómer. Orbitoesfenoides muy grande, ocupan $1 / 4$ del especio total de la órbita, con un elevado número de fenestras, en contacto en su parte anteroventral con el etmoides lateral, este último con una proyección cartilaginosa que alcanza el orbitoesfenoides, muy delgada. Paraesfenoides muy delgado, pasa por debajo de una terminación aguda posterior del vómer, no se encuentra en contacto con el orbitoesfenoides. Hiomandibular bifurcado con terminaciones agudas en la parte ventral, plegadas a la parte ventral del metapterigoides; cuadrado más desarrollado que en los demás congéneres (Londoño-Burbano 2009), con forámenes en toda su longitud, parte ventral unida al articular, el cual presenta una porción cartilaginosa anteriormente, por medio del cual se ve en contacto con el hueso del dentario. Mesetmoides con tres puntas redondas y de igual longitud, sin variación en su forma, no son independientes entre sí. Parte anterior del 
frontal se encuentra dividida entre dos terminaciones redondas. Fontanela y rinoesfenoides ausentes.

Seis supraneurales, el primero el más grande de los cinco restantes pero de menor tamaño que el presente en $S$. dariensis. Postcleitrum uno redondo y reducido, parte anterior en contacto con la parte posterior del supracleitrum el cual es muy largo. Postcleitrum dos muy largo, ancho y de forma irregular, su mitad anterior debajo del cleitrum, el cual es de forma triangular en su parte posterior, bifurcado en su parte anterior y en contacto en su parte dorsal. Postcleitrum tres muy delgado, en forma de espina, en inmediación anterior al postcleitrum dos. Hueso pélvico muy grueso y ancho, terminación anterior recta, sin procesos osificados en su longitud. Proceso isquial bifurcado en su parte posterior con ambas terminaciones agudas, con dos forámenes en la mitad de su longitud. 36 a 37 vértebras.

Color en alcohol. Superficie dorsal del hocico y la cabeza marrón, más claros a partir de una horizontal con la mitad del ojo hacia abajo. Dorso del cuerpo con bandas transversales en número de 8-10, de color marrón claro, se observan espacios de color amarillo pálido o blanco entre ellas, van desde el final del supraoccipital hasta el inicio del lóbulo caudal superior. Cabeza un poco más oscura arriba del ojo, más clara que la porción dorsal del cuerpo, debajo del ojo tiene una coloración blanca, sin manchas ni melanóforos; el cuerpo conserva el modelo de coloración que se presenta en la cabeza, arriba de la línea lateral es más oscuro, y con tendencia a marrón claro.

Se observan 6-8 bandas verticales de 2-3 escamas de ancho, en la parte lateral del cuerpo, arriba tocan la porción dorsal oscura y abajo llegan hasta dos escamas arriba de la base de las aletas ventrales, estas bandas algunas veces están divididas, forman dos manchas en la misma vertical, no se observa franja lateral sobre la línea lateral. Todas las aletas son hialinas, excepto la aleta caudal, que tiene los radios medios marrón, hasta el final de ellos. Parte ventral del cuerpo más clara que el resto, sin cromatóforos o manchas.

Caracteres sexuales secundarios. Tubérculos nupciales presentes sólo en machos mayores de $100 \mathrm{~mm}$ de LE, pequeños y de número muy reducido. Se observa una mayor cantidad al final del supraoccipital y lateralmente del mismo.

Distribución. Restringido a la cuenca alta del Río Cauca (Fig. 3).

\section{Saccodon dariensis \\ (Meek \& Hildebrand, 1913) \\ (Cuadros 1, 2 \& Fig. 2)}

Parodon dariensis Meek \& Hildebrand 1913:83 (descripción original; localidad tipo río Cupe, Panamá).

Apareiodon dariensis Eigenmann 1916:76 (diagnosis en clave). Eigenmann 1922: 111 (cuenca del río Tuyra; comentarios). Meek \& Hildebrand 1916:271 (río Cupe, Panamá; redescripción; foto en vista lateral). (Pavanelli 1999, 2003: sinonimia)

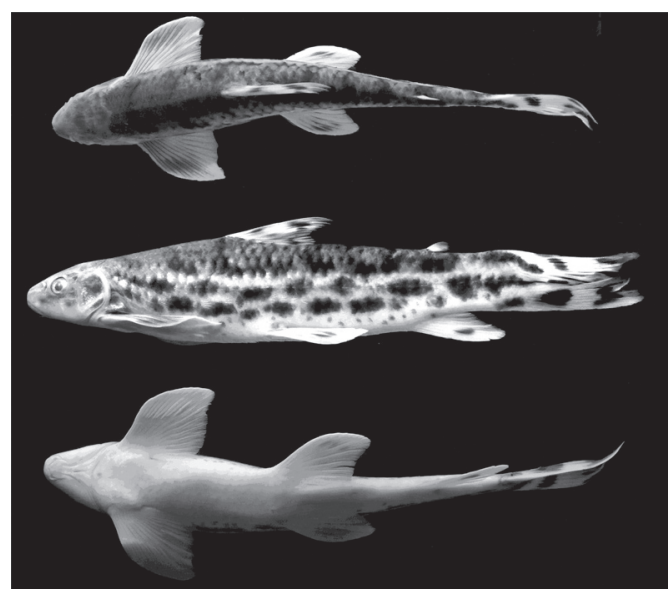

Fig. 2. Vistas dorsal, lateral y ventral de Saccodon dariensis, IUQ 2219, 130,98mm SL. Fotos por A. Londoño-Burbano (IUQ).

Fig. 2. Dorsal, lateral and ventral views of Saccodon dariensis, IUQ 2219, 130,98mm LE. Photos by A. Londoño-Burbano (IUQ). 


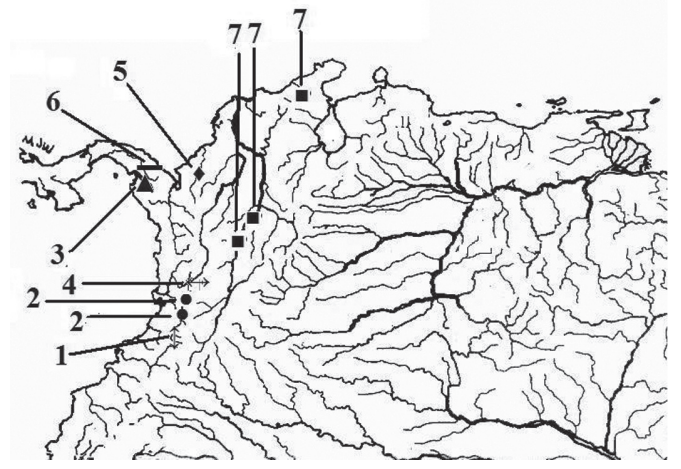

Fig. 3. Mapa de la distribución de Parodon caliensis y Saccodon dariensis; cada símbolo puede representar más de una localidad 1. Localidad tipo de Parodon caliensis (Río Cali); 2. Distribución de P. caliensis (Alto Cauca). 3. Localidad tipo de Saccodon dariensis (Río Cupe, Panamá); 4. Localidad tipo de S. caucae Schultz \& Miles, 1943 (Río Cauca); 5. Localidad tipo de Apareiodon brevipinnis Dahl, 1971 (Río Sinú); 6. Localidad tipo de A. compressus Breder Jr., 1925; 7. Distribución de S. dariensis (Río Magdalena, Cauca y vertiente Caribe-Guajira).

Fig. 3. Distribution of Parodon caliensis and Saccodon dariensis, each symbol may represent more than one locality. 1. Type locality of Parodon caliensis (Rio Cali). 2. Distribution of $P$. caliensis (Alto Cauca). 3. Type locality of Saccodon dariensis (Rio Cupe, Panamá). 4. Type locality of S. caucae Schultz \& Miles, 1943 (Rio Cauca). 5. Type locality of Apareiodon brevipinnis Dahl. 1971 (Rio Sinú). 6. Type locality of A. compressus Breder Jr., 1925. 7. Distribution of $S$. dariensis (Rio Magdalena, Cauca and Caribe-Guajira system).

Apareiodon compressus Breder Jr. 1925: 4 (descripción original; localidad tipo Darien, Panamá; ilustración en vista lateral y dientes). (Pavanelli 1999, 2003: sinonimia).

Saccodon dariensis Schultz \& Miles 1943:255 (diagnosis en clave; comentarios). Roberts 1974a: 316 (sinonímia; distribución; polimorfismo dentario; comentarios; fotos en vista lateral; ilustración de morfotipos). Roberts 1974b:416 (cuenca del río Bayano, Panamá; osteología). Géry 1977:207 (diagnosis en clave; foto en vista lateral; comentarios). Pavanelli 1999: 286-295 (revisión de la familia; sinonimias; claves y diagnosis; distribución; datos morfometría y merística). Pavanelli 2003:
48 (sinonimias; distribución; localización de material tipo; talla máxima). Ingenito 2008: 21 (análisis filogenético).

Saccodon caucae Schultz \& Miles 1943:262 (descripción original; localidad tipo: río Cauca; ilustración de la cabeza). Miles 1943:47 (cuenca del río Cauca; redescripción). Miles 1947:131 (cuenca del río Cauca; diagnosis en clave; comentarios). Wiley \& Collette 1970:166 (cuenca del río Magdalena; tubérculos nupciales). Dahl 1971:119 (cuenca del río Magdalena; comentarios). Pavanelli 1999, 2003 (sinonimia). Mojica et al. 2002: 212 (datos, endémica del alto Cauca, casi amenazada).

Apareiodon brevipinnis Dahl 1971:117 (descripción original; localidad tipo: río Verde, cuenca del río Sinú, Colombia; ilustración en vista lateral). Pavanelli 1999, 2003 (sinonimia).

\section{Material Examinado}

Holotipo. (FMNH 7587) 117.5mm. Panamá, vertiente Pacífica. Río Cupe, Cituro.

No tipos. Todos de Colombia: IUQ 1673 (3: 58.43-65.83mm LE), cuenca del Rio Cauca. Río Roble, afluente rio la Vieja en la reserva natural el Ocaso, $100 \mathrm{~m}$ abajo del puente peatonal en Playa Azul, Municipio de Quimbaya, departamento del Quindío 43 ‘ $68^{\prime}$ ” N y 75॰52'81' W, 19-VIII-2007. IUQ 1672 (2: 37.09 y $38.13 \mathrm{~mm}$ LE), cuenca Río Cauca. Quebrada Las Cañas afluente Río Cauca, vía a Zarzal, Valle $4^{\circ} 21^{\prime} 09^{\prime \prime} \mathrm{N}$ y $76^{\circ} 04^{\prime} 11^{\prime \prime} \mathrm{W}$, Municipio de Zarzal, 6-VIII-2007. IUQ 1954 (2: 32.40 y 38.96mm LE), cuenca del Río Cauca. Quebrada Las Cañas afluente del Río Cauca en la vía Zarzal-La Paila, departamento del Valle del Cauca, Municipio de Zarzal, 16-II-2008. IUQ 2222, ex CP-UCO 336 (2: 134.39 y $105.63 \mathrm{~mm}$ LE), cuenca del Río Magdalena, Quebrada Arenal, San Rafael, departamento de Antioquia Municipio de San Rafael, 9-IX-2006. IUQ 2219, ex CP-UCO 4 (2: 96.71 y $130.98 \mathrm{~mm}$ LE), cuenca del Río Magdalena. Rio La Miel, departamento de Caldas, Municipio Samaná, desembocadura Río Tasajos en la Miel 
Municipio de Samaná, 2-II-2006. IUQ 1219 (1: 108.89mm LE), cuenca del Río Cauca. Río la Vieja, cuenca del Alto Cauca, departamento del Quindío, sin fecha. IUQ 896 (1: $56.05 \mathrm{~mm}$ LE), cuenca del Río Cauca. R. la Vieja en Puerto Alejandría, departamento del Quindío, municipio de Quimbaya, 7 -VII-1993. IUQ 777 (1: 143.76mm LE) cuenca del Río Cauca. R. La Vieja, Alambrado, departamento del Quindío, Municipio de La Tebaida, 11-VI-1991.

English diagnosis. S. dariensis is distinguished from $S$. wagneri from the presence of two to three rows of separated round spots along the flanks of the body (vs. presence of a lateral stripe); from $S$. terminalis by having a ventral mouth (vs. terminal mouth); and from both species by having 12 circum-peduncle scales (vs. 16 scales) (see diagnosis of P. caliensis for differences between these two species).

Diagnosis. $S$. dariensis se diferencia de S. wagneri por presentar de dos a tres filas de manchas redondas separadas entre sí en la parte lateral del cuerpo (contra presencia de franja lateral); de $S$. terminalis por presentar boca ventral (contra boca casi terminal). De ambos congéneres se diferencia por tener 12 escamas circumpedunculares (contra 16 escamas). (Véase diagnosis de $P$. caliensis para diferencias entre ambas especies).

Descripción. Datos morfométricos y merísticos se consignaron en los cuadros 1 y 2 , respectivamente. Cuerpo rollizo. Perfil dorsal del cuerpo recto desde el hocico hasta el final del supraoccipital, a partir de allí convexo hasta el inicio de la aleta dorsal y recto hasta el inicio del lóbulo caudal superior. Perfil ventral del cuerpo recto desde el hocico hasta el inicio de la aleta anal, desde allí cóncavo hasta el inicio del lóbulo caudal inferior. Istmo en una vertical con la parte posterior del supraoccipital. Membranas branquiales unidas entre si y libres del istmo. Ojos laterales. Narinas a la altura del ojo, sin membrana de piel que sobresalga ni que divida la narina en dos partes, redondos.
Boca ventral, labio superior ausente; pedúnculo caudal comprimido.

El número de cúspides de los dientes del premaxilar es variable dado el polimorfismo presentado por esta especie: su número varía entre cero y 20 cúspides. Los dientes se encuentran en diferentes posiciones: en línea recta, sobreponiéndose uno sobre el otro o en forma de abanico siendo de mayor tamaño los dientes en esta forma. El número de dientes en la misma región es entre tres y cuatro. Mandíbula inferior sin dientes, el labio inferior recto, convexo o con tres o cinco lóbulos enmarcando la posición de los dientes del premaxilar. Aletas pectorales truncadas, más largas y anchas que en P. caliensis (Cuadro 1), no alcanzan las aletas ventrales, y su inicio en una vertical trazada desde la parte posterior del supraoccipital a la parte ventral del cuerpo, el cuarto y quinto radio ramificado mas alargados que el resto. Aletas ventrales truncadas, siempre sobrepasan el poro genital por dos o tres escamas, su inicio en una vertical del final de la base de la aleta dorsal hacia la parte ventral del cuerpo, con el cuarto radio ramificado más alargado que el resto. Aleta anal truncada, alcanza el lóbulo caudal inferior, truncada, su inicio en una vertical trazada desde una escama anterior al comienzo de la aleta adiposa hacia la parte dorsal del cuerpo, los dos primeros radios ramificados más alargados que el resto. Aleta dorsal truncada, con los primeros radios ramificados más alargados que el resto, no llega hasta la adiposa por 9-10 escamas. Comienzo de la aleta adiposa en una vertical trazada desde el final de la base de la aleta anal hacia la parte dorsal del cuerpo. Aleta caudal bilobulada, lóbulo inferior más largo que el superior, con los radios medios de color negro hasta el final de ellos y entre tres a cuatro manchas negras en ambos lóbulos. Base de la aleta caudal desnuda. Línea lateral completa con 38-41 escamas con poros, no extendida sobre la base de la aleta caudal. Escamas dispuestas regularmente en el área predorsal y ventral. Escama axilar presente de una escama de longitud.

Observaciones osteológicas. Antorbital triangular en contacto con el supraorbital y en 
contacto en la parte ventral con el infraorbital uno. Seis infraorbitales, el canal laterosensorial pasa por todos ellos. Supraorbital presente, se observa algunas veces más ancho en la parte anterior o igual de ancho en toda su longitud, en contacto posterior con el infraorbital seis. Nasal en empalme posterior con el frontal. Premaxilar muy desarrollado, redondo con una hilera de dientes de reposición, observándose un diente por cada cavidad. Maxilar cóncavo con una terminación muy aguda en su parte posterior, no se observan dientes de reposición. La porción dorsal del postemporal no toca la parte posterior del supraoccipital, antero-ventralmente en contacto con el extraescapular. Pterótico llega a la parte anterior del extraescapular y dorsalmente al parietal. Metapterigoides en contacto dorsalmente con el mesopterigoides y anteriormente unido por una porción cartilaginosa al ectopterigoides, este último muy alargado, con su porción anterior más ancha que la posterior, con una terminación redonda. Palatino unido posteriormente con el ectopterigoides, su porción anterior es redonda, mucho más ancha que la porción posterior, con fenestras, esta porción se encuentra exactamente encima del vómer, el cual es una estructura muy grande y gruesa de forma irregular. Orbitoesfenoides con numerosas fenestras, en contacto antero-ventral con el etmoides lateral. Paraesfenoides muy largo y delgado, con bordes rectos, por medio de una porción cartilaginosa se encuentra unido dorsalmente al orbitoesfenoides y ventralmente al vómer. Hiomandibular con su porción anterior bífida, estando el metapterigoides insertado en esta concavidad, su parte posterior con una terminación redonda encontrándose sobre el opérculo. Cuadrado en contacto anteriormente con el articular, el cuadrado es una estructura pequeña recta ventralmente, cóncava dorsalmente y redonda anteriormente. El articular es una estructura alargada y ancha, anteriormente se encuentra bajo el maxilar, con un foramen en su porción más anterior, con tres proyecciones dorsales, siendo la más anterior la que más sobresale, recto ventralmente, estando en contacto en esta misma porción con el dentario. Mesetmoides con tres puntas anteriormente, la de la mitad más delgada y larga, posteriormente con dos proyecciones laterales bífidas, ambas independientes de la proyección central sin entrar en contacto con la parte anterior del frontal, el cual es bilateral horizontalmente, anteriormente con dos terminaciones redondas, con dos crestas muy gruesas, unidas medial y posteriormente; fontanela y rinoesfenoides ausentes.

De seis a siete supraneurales, el primero muy grande, robusto, de forma irregular, mientras que el resto son delgados y de forma alargada, en contacto ventralmente con la primera espina hemal. Postcleitrum uno muy grueso en contacto ventralmente con el cleitrum, posteroventralmente con el postcleitrum dos. Postcleitrum dos en contacto con el cleitrum. Postcleitrum tres muy delgado y largo proyectado ventralmente, en contacto dorsalmente con el postcleitrum dos. Pterigióforos de la aleta dorsal insertados entre las espinas hemales 8-16. Hueso pélvico muy grueso, con una terminación muy aguda y extensa, con proyecciones a lo largo de toda la estructura, sin forámenes. Proceso isquial delgado, con una protuberancia ventral, con una terminación menos aguda que la observada en el hueso pélvico. 37-38 vértebras.

Color en vivo. Área dorsal verde oscuro; fondo del cuerpo blanco, plateado más pronunciado en la parte ventral del cuerpo. En la parte dorsal se observan ocho bandas transversales oscuras que alcanzan en la parte lateral nueve manchas oscuras. Aletas pectorales y pélvicas amarillo claro; dorsal y anal grises; caudal con dos manchas oscuras en la parte media de los lóbulos.

Color en alcohol. Superficie dorsal del hocico y la cabeza negra, de igual color con algunas manchas amarillas en los infraorbitales dos y tres, en algunos ejemplares esta porción se observó más clara, blanca o amarilla. Dorso del cuerpo negro, con 8-10 bandas transversales ubicadas desde una escama posterior al final del supraoccipital hasta el inicio del lóbulo caudal superior. La porción lateral del cuerpo se observa totalmente negra desde la porción 
dorsal del opérculo hacia arriba, debajo de éste se observa el fondo blanco o amarillo.

Se observan de dos a tres filas de manchas negras redondas o alargadas independientes, de longitud y número variables en la parte lateral del cuerpo, no se observa banda lateral sobre la línea lateral. Aletas pectorales con una mancha negra muy larga en los primeros seis radios desde la parte más distal de los mismos hasta el inicio de la aleta. Aletas ventrales con una mancha negra de forma irregular en la mitad de los tres primeros radios ramificados. Aleta anal con una mancha negra pequeña, redonda en la mitad de los dos primeros radios ramificados sin extenderse hasta el inicio de la aleta. Aleta dorsal con dos o tres manchas negras de forma irregular, ubicándose en una porción próxima a la base de la aleta, algunas veces en la mitad de su longitud y otra alcanzando la parte más distal de la misma. Aleta caudal con los radios medio negros hasta el final de los mismos, con manchas negras en ambos lóbulos en la base y en la parte más distal; aleta adiposa hialina.

Caracteres sexuales secundarios. No se observaron tubérculos nupciales, posiblemente debido a la época de colecta de los ejemplares evaluados.
Distribución. Sistema del río Magdalena, Cauca, vertiente Caribe-Guajira en Colombia y parte de Panamá: río Cupe (localidad tipo) y río Tuquesa (Fig. 3).

\section{DISCUSIÓN}

$P$. caliensis es diferenciado de $S$. dariensis por su modelo de coloración, y morfometría corporal, siendo las variables que más aportaron a esta diferenciación: la longitud de la mandíbula inferior, longitud del hocico, la distancia desde el istmo a las aletas pectorales, desde las aletas ventrales a la aleta anal y la distancia desde el final de la base de la aleta dorsal a la aleta adiposa, las cuales con ayuda de un análisis de componentes principales (ACP) lograron ilustrar diferencias sutiles entre ambos taxones, donde las dos primeras componentes explican el $71.60 \%$ de la variación (Figs. 4 y 5). La longitud del lóbulo caudal inferior en $S$. dariensis es mayor que el superior, caso contrario a lo que ocurre en $P$. caliensis y en todas las especies del género Parodon con excepción de $P$. bifasciatus. De igual forma, conteos merísticos como el número de escamas post anales permitió separar ambos taxones, además de otros conteos (Cuadro 2).

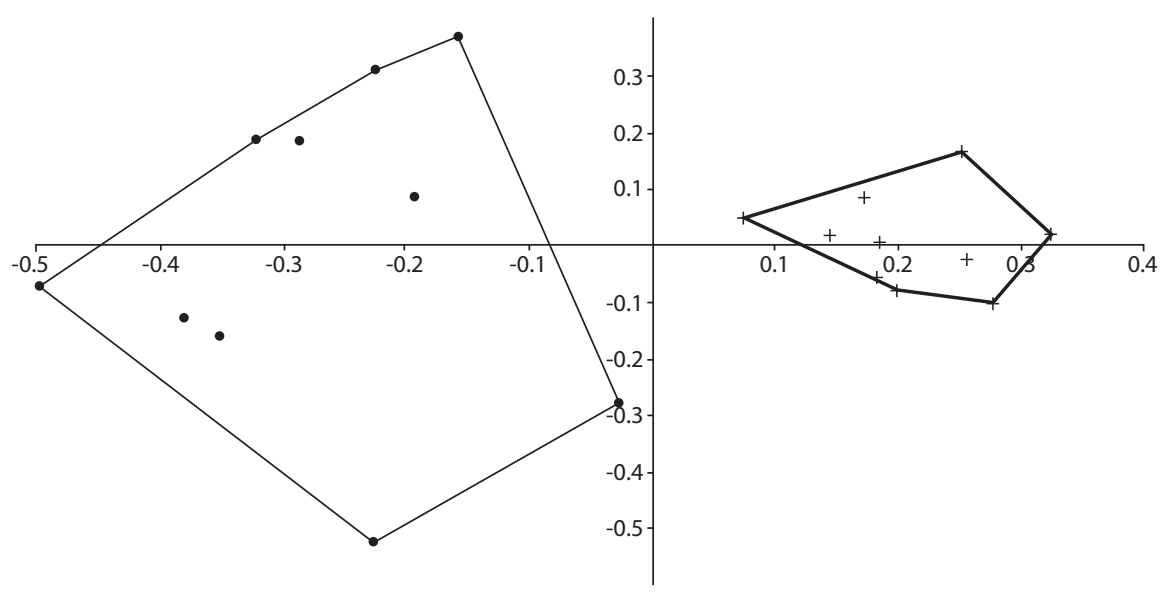

Fig. 4. Análisis de componentes principales (ACP) de la variación morfológica de $(+)$ : Parodon caliensis y $(\bullet)$ : Saccodon dariensis. La primera, segunda y tercera componente explican el $71.60 \%$ de la variación.

Fig. 4. Principal component analysis (PCA) of the morphologic variation of $(+)$ : Parodon caliensis and $(\bullet)$ : Saccodon dariensis. The first, second and third component explains the $71.60 \%$ of the variation. 


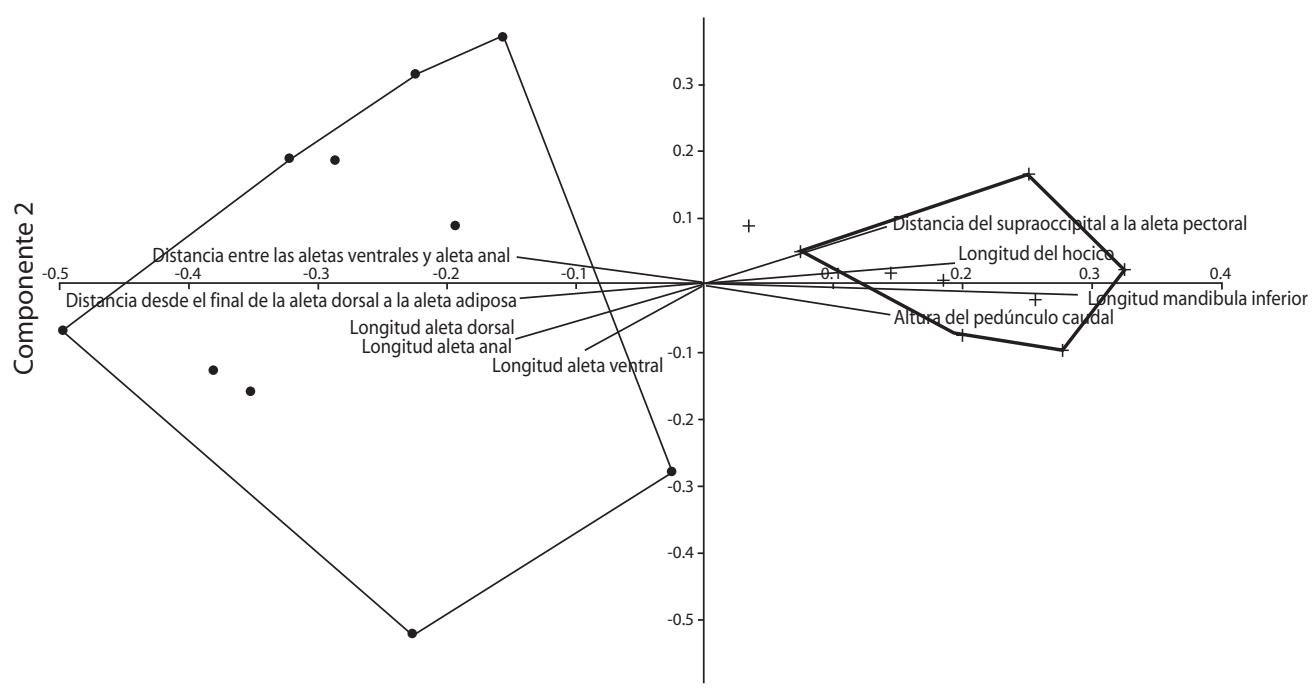

Componente 1

Fig. 5. Variación morfológica de (+): Parodon caliensis y (•): Saccodon dariensis.

Fig. 5. Morphologic variation of (+): Parodon caliensis and (•): Saccodon dariensis.

Según Pavanelli $(1999,2003)$ Apareiodon brevipinnis Dahl 1971 es un sinónimo de $S$. dariensis, sin embargo, cabe resaltar en especial los conteos con respecto a las aletas pectorales ofrecidas por el autor: "i, 14- i, 15" difiriendo con uno de los caracteres diagnósticos para el género Saccodon el cual posee dos radios no ramificados en las aletas pectorales. El holotipo de esta especie se encuentra desaparecido (único ejemplar citado bajo dicho nombre), lo cual dificulta su evaluación, además, no se encontraron registros de ejemplares de $S$. dariensis, $P$. caliensis u otro integrante de la familia Parodontidae provenientes del río Verde, afluente del río Sinú (localidad tipo de A. brevipinnis) o afluentes del mismo, lo cual hace necesaria la valoración de dicha cuenca para llegar a una conclusión definitiva acerca de la especie descrita por Dahl (op. cit). De igual manera, Breder Jr. (1925) describió $A$. compressus proveniente del río Turquesa, Darien, Panamá; se examinó fotografía del holotipo (AMNH 8408; http://entheros.amnh.org/ $\mathrm{db} /$ emuwebamnh/pages/common/imagedisplay.php) el cual mide $23 \mathrm{~mm}$ de LE y pasados más de 80 años desde su colecta se observa deteriorado, con las aletas destruidas casi en su totalidad y ausencia casi total de escamas, sin embargo, se pueden apreciar algunas características con respecto a su coloración la cual está compuesta por tres grandes manchas que van desde la parte dorsal del cuerpo hasta la parte ventral del mismo, sin observarse el modelo de coloración típico de $S$. dariensis que está compuesto por una serie de tres filas de manchas redondas pequeñas (aún en ejemplares juveniles); Breder Jr. (op. cit.) en su descripción original citó: "sides with a single dark lateral band following the lateral line" describen la banda lateral propia de la gran mayoría de especies pertenecientes al género Parodon y aún a la familia Parodontidae en su totalidad pero ausente en $S$. dariensis. En contraste con la mención de la mandíbula inferior sin dientes (Breder Jr., op. cit.) (característica propia del género Saccodon); el ejemplar examinado es el único relacionado con el nombre propuesto por el autor, siendo la localidad tipo del mismo compartida con $S$. dariensis de acuerdo a lo anotado por Pavanelli 
(2003). A pesar del estado juvenil del holotipo de la especie y su deterioro, se mantiene la sinonimia de éste con $S$. dariensis, siendo ésta la única especie reportada para Panamá en Centro América perteneciente al género Saccodon y aún a la familia Parodontidae.

A partir de la evaluación de la disposición dentaria en $S$. dariensis se encontraron cuatro tipos de morfos descritas por Roberts (1974a), ausente el morfo $\mathrm{V}$ y la transición entre los morfos I a IV (Roberts, op. cit.): Los morfos I, II y III se observaron más constantes en localidades a la altura del departamento del Quindío (Alto Cauca) y en ejemplares de menos de $85 \mathrm{~mm}$ de LE, mientras que el morfo IV se observó casi exclusivamente en ejemplares presentes a la altura del departamento del Valle del Cauca (Alto Cauca) $\mathrm{y}$ en individuos mayores a $100 \mathrm{~mm}$ LE, esto puede estar relacionado al tipo de ambientes, sustratos y recursos alimenticios encontrados en las diferentes localidades las cuales pueden ser explotadas de una manera más eficiente de acuerdo a la disposición dentaria presentada por los ejemplares; de igual forma, y de acuerdo a lo descrito por Roberts (op. cit.) el número elevado de adultos con este tipo de dentición puede estar relacionado también con cambios ontogénicos.

Debido al impacto antrópico que ha sufrido el río Cauca en todos sus hábitats tanto por el vertimiento de desechos domésticos e industriales como por la constante remoción de sustrato (Patiño1991, Zúñiga et al. 1994), se ha observado, tanto en campo como en registros de colecciones, la disminución y posible desaparición de $P$. caliensis, dado que el registro más reciente que se tiene de ejemplares capturados data de 1999 (véase material examinado), sin embargo, se hace necesario el inventario en afluentes cercanos al río Cali (localidad tipo de P. caliensis) ya que dicho sistema se encuentra en condiciones anóxicas por encontrarse dentro del casco urbano, lo cual dificulta un estudio de poblaciones que podrían estar allí presentes, siendo las especies de la familia Parodontidae propias de aguas rápidas y oxigenadas, condiciones ausentes en dicho río.

\section{AGRADECIMIENTOS}

Se recibió financiación de La Universidad del Quindío-la Rectoría de Alfonso Londoño y de la Vicerrectoria de investigaciones, a través del fondo para semilleros de investigación y de varios proyectos (a CR-V). Por el préstamo de material y envío de fotografías de material tipo: Barbara Brown y Scott Schaefer (AMNH), James Maclaine (BMNH), David Catania y Mysi Hoang (CAS), Gabriel Roldán, María Isabel Ríos y Luisa F. Álvarez A. (CP-UCO), Mary A. Rogers, Kevin Swagel y Phil Willink (FMNH), Raúl Ríos y Germán Parra (IMCN), Andrew Williston y Karsten Hartel (MCZ), y Carla S. Pavanelli (NUP). Por sus comentarios sobre parodóntidos a Carla S. Pavanelli (NUP), William Eschmeyer (CAS), Carl Ferraris Jr. (CAS) y Martine Desoutter (MNHN). Tres revisores anónimos efectuaron correcciones y críticas muy constructivas al artículo.

\section{RESUMEN}

La familia Parodontidae es un grupo de peces Characiformes distribuidos a través de Sur América y parte de Panamá, contiene tres géneros: Apareiodon Eigenmann 1916, Parodon Valenciennes 1849 y Saccodon Kner 1863, con 28 especies reconocidas y dos géneros válidos: Parodon y Saccodon. Se realizó la redescripción de Parodon caliensis y Saccodon dariensis a partir de material tipo, y topotípico de Colombia. Se valoraron caracteres morfológicos, merísticos osteológicos y de coloración, encontrándose diferencias significativas entre ellos. Se halló una amplia distribución para $S$. dariensis, mientras $P$. caliensis se restringe al Alto Cauca. Tres especies son consideradas aquí como sinonimias de $S$. dariensis: Apareiodon dariensis, A. compressus y $S$. caucae. Se describe la presencia de dimorfismo sexual en ambas especies.

Palabras clave: Parodon, Saccodon, taxonomía, Colombia, pez tropical.

\section{REFERENCIAS}

Boulenger, G.A. 1895.Description of a new Characinoid fish of the Genus Parodon. Ann. Mag. Nat. Hist. 16: 480 .

Breder Jr., C.M. 1925. New loricariate, characin and poeciliid fishes from the Rio Chucunaque, Panama. Amer. Mus. Nov. 180: 1-9. 
Burnaby, T.P. 1966. Growth-invariant discriminant functions and generalized distances. Biometrics 22: 96-110.

Dahl, G. 1971. Los peces del norte de Colombia. Instituto de Desarrollo de los Recursos Naturales Renovables/ INDERENA/Ministerio de Agricultura, Arco, Bogotá, D.C., Colombia.

Eigenmann, C.H. 1916. On Apareiodon, a new genus of characid fishes. Ann. Carn. Mus. 10: 71-76.

Eigenmann, C.H. 1922. The fishes of Western South America, Part I. The fresh-water fishes of Northwestern South America, including Colombia, Panama, and the pacific slopes of Ecuador and Peru, together with an appendix upon the fishes of the Rio Meta in Colombia. Mem. Carnnegie Mus. 9: 1-348.

Géry, J. 1977. Characoids of the world. THF, Neptune City, New Jersey, EEUU.

Ingenito, L.F.S. 2008. Análise Filogenética da Família Parodontidae (Teleostei, Characiformes). Tesis Doctorado, Universidade Federal do Rio de Janeiro, Brasil.

Londoño-Burbano, A. 2009. Revisión taxonómica para Colombia de las especies del género Parodon (Characiformes: Parodontidae). Trabajo de grado (B.Sc.), Universidad del Quindío, Armenia, Colombia.

Maldonado, J.A., A. Ortega-Lara, S. Usma, V.G. Galvis, F.A. Villa-Navarro, S. Prada \& R. Ardila. 2005. Peces de los Andes de Colombia. Instituto de Investigación de Recursos Biológicos "Alexander von Humboldt", Ramos López, Bogotá, D.C., Colombia.

Meek, S.E. \& S.F. Hildebrand. 1913. New species of fishes from Panama. Field Mus. Nat. Hist. Zool. 10: 77-91.

Meek, S.E. \& S.F. Hildebrand. 1916. The fishes of the fresh waters of Panama. Field Mus. Nat. Hist. Zool. 10: $217-374$.

Miles, C. 1943. Estudio económico y ecológico de los peces de agua dulce del Valle del Cauca. Secretaría de Agricultura y Fomento del Departamento del Valle del Cauca, Cali, Colombia.

Miles, C. 1947. Los peces del rio Magdalena: ("A field book of Magdalena fishes"). El Gráfico, Bogotá, Colombia.

Mojica, J.I., C. Castellanos, S. Usma, \& R. Álvarez. 2002. Libro Rojo de peces dulceacuícolas de Colombia. Instituto de Ciencias Naturales, Universidad Nacional de Colombia, Ministerio de Medio Ambiente, Panamericana, Bogotá, Colombia.

Patiño, A. 1991. Ecología y compromiso social: itinerario de una lucha. Cerec, Bogotá, D.C., Colombia.
Pavanelli, C.S. 1999. Revisão taxonômica da família Parodontidae (Ostariophysi: Characiformes). Tesis Doctorado, Universidade Federal de São Carlos, São Carlos, Brasil.

Pavanelli, C.S. 2003. Family Parodontidae (Parodontids) In Checklist of the freshwater fishes of South y Central America. R.E. Reis, S.O. Kullander and C. Ferraris, Jr. (eds.). EDIPUCRS, Porto Alegre, Brazil, p. 46-50.

Pavanelli, C.S. \& H.A. Britski. 2003. Apareiodon Eigenmann, 1916 (Teleostei, Characiformes), from the Tocantins-Araguaia Basin, with description of three New Species. Copeia 2: 337-348.

Roberts, T.R. 1974a. Dental polymorphism and systematics in Saccodon, Neotropical genus of freshwater fishes (Parodontidae, Characoidei). J. Zool. Soc. London 173: 303-321.

Roberts, T.R. 1974b. Osteology and classification of the Neotropical characoid fishes of the families Hemiodontidae (including Anodontidae) and Parodontidae. Bull. Mus. Comp. Zool. 146: 411-472.

Ruiz-C., R.I. \& C. Román-Valencia. 2006. Osteología de Astyanax aurocaudatus Eigenmann, 1913 (Pisces, Characidae), con notas sobre la validez de Carlastyanax Géry, 1972. Anim. Biodivers. Conserv. 29: 49-64.

Schultz, L.P. \& C. Miles. 1943. Descriptions of a new genus and a new species of Parodontinae, characinid fishes from South America. J. Wash. Acad. Sci. 8: 251-255.

Song, J. \& L.R. Parenti. 1995. Clearing and staining whole fish specimens for simultaneous demonstration of bone, cartilage and nerves. Copeia 1: 114-118.

Taylor, W.R. \& G.C. Van Dyke. 1985. Revised procedures for staining and clearing small fishes and other vertebrates for bone and cartilage study. Cybium 9: 107-119.

Weitzman, S.H. 1962. The osteology of Brycon meeki, a generalized characid fish, with an osteological definition of the family. Stanford Ichthyol. Bull. 8: 1-77.

Wiley, M.L. \& B.B. Collette. 1970. Breeding tubercles and contact organs in fishes: their occurrence, structure and significance. Bull. Amer. Mus. Nat. Hist. 143: 146-215.

Zúñiga, M.C., A.M. Rojas \& C. Serrato. 1994. Interrelación de indicadores ambientales de calidad de cuerpos de aguas superficiales del Valle del Cauca. Rev. Col. Ento. 20: 124-130. 